\title{
Premiers RÉSUltats D'UNE EXPÉRIENCE DE SÉLECTION SUR LA PROI,IFICITÉ DES TRUIES
}

\author{
L. OILIVIER et C. LEGAULT. - Station de Génétique quantitative et appliquée, \\ C.N.R.Z., 78-Jouy-en-Josas
}

Une expérience de sélection a été entreprise au domaine de Galle (18-Avord), afin d'estimer les possibilités d'amélioration génétique de la prolificité des truies dans un troupeau fermé. Le critère de sélection (P) est le nombre total de porcelets normaux (vivants et morts) nés dans les deux premières portées de chaque truie. Les autres performances d'élevage ainsi que les caractéristiques d'engraissement et de carcasse sont également contrôlées à chaque génération. Enfin, le taux d'ovulation et la mortalité embryonnaire sont estimés sur un échantillon de truies abattues vers le $30^{\mathrm{e}}$ jour de leur $3^{\mathrm{e}}$ gestation.

Cent vingt truies et 10 verrats $\left(F_{0}\right)$ ont été achetés en octobre 1965 dans 12 élevages inscrits au livre généalogique de la race Large-White et les premières mises-bas ont eu lieu au printemps de l'année suivante; $19,33 \pm 1,18$ et $15,13 \pm 1,12$ porcelets ont été respectivement mis bas et sevrés par ces truies dans leurs deux premières portées et l'examen du tractus génital de 30 de ces animaux montrait $17,32 \pm 0,98$ corps jaunes et une mortalité embryonnaire de $36,05 \pm 4,22 \%$.

Deux lignées ont été constituées à l'automne 1966; alors que dans la lignée témoin (T) le choix des reproducteurs est fait au hasard (10 $\sigma^{\top} \sigma^{\star}$ et 40 qq $)$, dans la lignée sélectionnée (S), il est fait dans les premières portées des truies les plus prolifiques (10 ठరో et 80 q\%). Dans les deux cas il s'agit d'un choix effectué à l'intérieur de familles de demi-frères et demi-sceurs. Les résultats de la $F_{1}$, concernant respectivement le nombre de porcelets nés, sevrés, de corps jaunes et la mortalité embryonnaire sont les suivants :

Lignée $S: 21,78 \pm 1,32 ; 14,68 \pm 1,20 ; 17,69 \pm 1,19$ et $22,09 \pm 4,00 \%$.

Lignée $T: 20,87 \pm 1,94 ; 14,39 \pm 1,92 ; 17,38 \pm 1,35$ et $19,81 \pm 5,52 \%$.

Pour une différentielle de sélection de 1,97 porcelet, le progrès génétique réalisé est de 0,91 porcelet alors qu'en prenant 0,20 comme estimation de l'héritabilité de $(P)$ le progrès génétique théorique était de 0,20 porcelet.

\section{DIFFF́RENCE FNTRE, SOUCHES DE VOLAILLES POUR LA RÉPONSE DES JEUNES}

\section{A Une avitaminose $A$}

Jacqueline Prod'homme ét P. Ḿ́rat (*)

(*) Station centrale de Génétique animale, C.N.R.Z., 78-Jouy-en-Josas.

Des observations antérieures sur des poussins Faverolles en cages, recevant à 12 jours une dose unique de vitamine $A$, puis un aliment carencé en cet élément, montraient pour ces animaux une survie de plus longue durée que celle obtenue par d'autres auteurs, avec un avantage apparent aux plumages saumonés "clairs " comparés aux "foncés ".

La comparaison présente porte sur une population de Faverolles saumonées et une souche " synthétique " (L22) obtenue par croisement de Leghorns et d'autres races. Au total, 288 poussins sexés, mis en cages individuelles dès l'éclosion en janvier 1966 (72 par sexe pour chaque lignée) ont reçu pour moitié un aliment carencé en vitamine $A$ et caroténoïdes, pour moitié le même aliment supplémenté en ces facteurs, dont la teneur était contrôlée en début et en fin d'expérience.

La mortalité de la première semaine (considérée comme préexpérimentale) était assez élevée (20,8\% globalement) du fait des difficultés d'élevage individuel au départ, mais ne différait pas entre souches. Par la suite, cette mortalité - faible en régime non carencé - était plus précoce pour la souche $L 22$ que pour la Faverolles en régime carencé (en fin de $5^{\mathrm{e}}$ semaine, $50,0 \%$ contre $28,6 \%$ chez les mâles, $20,0 \%$ contre $7,1 \%$ chez les femelles). A 50 jours, elle se trouvait à peu 